\title{
National Guard Couples Communicating During Deployment: The Challenge of Effective Connection
}

\author{
Adrian J. Blow ${ }^{1} \cdot$ Adam M. Farero $^{1}$ (D) $\cdot$ Lisa (Gorman) Ufer ${ }^{2} \cdot$ Michelle Kees $^{3} \cdot$ Danielle Guty $^{2}$ \\ Accepted: 1 June 2021 / Published online: 6 June 2021 \\ (c) The Author(s), under exclusive licence to Springer Science+Business Media, LLC, part of Springer Nature 2021
}

\begin{abstract}
Communication during a combat deployment has changed significantly in current times. Couples can now communicate with each other frequently and through multiple modes. Despite this greater availability of communication options, there remain unanswered questions related to how healthy deployment communication is best achieved between couples, particularly regarding navigating the uncertainty of deployment. In this qualitative study, we report on the experiences of 31 National Guard couples who endured a combat deployment in support of Operation Enduring Freedom. Couples were interviewed three months after the soldier returned from deployment. Through the lens of relational turbulence theory, we provide a conceptual framework related to effective and non-effective deployment communication, along with structural communication barriers in the military that may negatively affect the mental well-being of partners. Finally, we provide recommendations to guide couples through these difficult deployments.
\end{abstract}

Keywords Military $\cdot$ Deployment $\cdot$ Military couples $\cdot$ Military communication

\section{Introduction}

Recent advances in technology have made real-time deployment communication an option for military couples. It is now possible for service members to communicate via video, email, phone, instant messaging, web cams, and variations of social media, in addition to more traditional formats such as letter writing. These developments have made regular and more interactive communication with at-home intimate partners during deployment a reality for service members, allowing for ongoing connection throughout the deployment (Cigrang et al., 2014; Wong \& Gerras 2006).

However, communication between couples during a military deployment is complex, and may lead to a variety of varying outcomes for both service members and their partners. Regular, positive communication can be a strength to

Adam M. Farero

fareroad@msu.edu

1 Department of Human Development and Family Studies, Michigan State University, East Lansing, MI, USA

2 Michigan Public Health Institute, Okemos, MI, USA

3 Department of Psychiatry, University of Michigan, Ann Arbor, MI, USA couples, and may include increased peace of mind, lowered PTSD risk for the service member, and a greater sense of support, trust, and intimacy in the relationship, which may facilitate greater resilience for all throughout deployment (Baptist et al., 2011; Carter et al., 2011; Merolla, 2010; Moelker \& van der Kloet, 2006). Conversely, the ability to communicate with family members on a daily basis can be distracting and can remind service members of what they are missing out on at home, while also creating an increased pressure to make daily contact (Cigrang et al., 2014; Durham, 2010). In extreme cases, breakdown in intimate or family relationships has been identified as the highest primary stressor related to soldier suicide during deployment (Martin et al., 2009; Warner et al., 2007), implying that the consequences of high-conflict communication during deployment may be severe.

This variability in the outcome of couple communication highlights the need for the ongoing, more nuanced study of what factors contribute to healthy, effective communication between partners during a deployment (Knobloch et al., 2018). In this paper we describe the communication experiences of 31 National Guard couples who together endured a combat deployment during Operation Enduring Freedom. Through qualitative methods we examined military couples' experiences of uncertainty in deployment-related 
communication, as well as their attempts to be planful and intentional in their efforts to stay in touch.

\section{Theoretical Framework}

In examining the nuances of couple communication during deployment, we draw on Relational Turbulence Theory (RTT; Solomon et al., 2016). According to RTT, transition periods are uniquely stressful for couples, and may contribute to relational turbulence, or intense emotional, cognitive, and communicative reactions to the context of the relationship. Relational uncertainty and partner interference are two mechanisms by which relational turbulence is theorized to occur during such transitions, and both have been applied to military couples' challenges during reintegration (Knobloch \& Theiss, 2014; Knobloch et al., 2013; Theiss \& Knobloch, 2013). With relational uncertainty, a partner questions their own or their partner's commitment, or the future of the relationship. In partner interference, an individual is perceived as disrupting their partner's efforts to accomplish their own goals. Both circumstances influence the ways in which couples communicate with one another, and can create greater relationship turmoil. Relational turbulence through these two mechanisms may be at play within military couples' relationships not only at reintegration, but also during the deployment itself. Such a phenomenon merits exploration.

\section{Potential Contributors to Relational Turbulence during Deployment}

The unpredictable nature of deployment communication is a serious threat to military couples' sense of stability in their relationship. Despite the convenience of real-time communication, it can have serious drawbacks. It can be difficult to coordinate a call time given time-zone differences and soldiers' unpredictable deployment schedules. Additionally, there is the potential for loss of connection or missed calls, both of which can be a source of stress or anxiety, particularly for the at-home partner (Sayers et al., 2018). In fact, there is evidence that delayed modalities, such as letters, packages, and emails, are tied to better outcomes, including soldier mental health post-deployment (Carter et al., 2011). As the use of real-time communication during deployment is relatively recent, there is a need for further study of how couples handle its potential stressors and unpredictability.

Another potential contributor to relational turbulence during deployment communication is the tendency for partners to withhold information from one another. In a recent qualitative study of veterans and partners, nearly half of participants reported avoiding discussing certain topics, for a variety of reasons (Sayers et al., 2018). An inability to speak openly about mission details, whether due to confidentiality or a desire to censor traumatic events, can exacerbate stress experienced by soldiers and leave non-deployed partners feeling unsure how to provide adequate support (Baptist et al., 2011; Durham, 2010; Wong \& Gerras 2006). This withholding may leave a partner feeling detached, particularly in situations where relationship satisfaction is low (Sayers et al., 2018). Fear of adding to the burden of the deployed soldier can also lead a partner to withhold stressful news, known as protective buffering, which may result in worse mental health for the at-home partner (Joseph \& Afifi, 2010). Despite the potentially detrimental effects of withholding information from one another, the outcome may be better when soldier and partner manage expectations and agree ahead of time which information to share right away, and which can wait until the soldier returns home (Sayers et al., 2018). Further examination of what this planning looks like in military couples and its potential benefits is needed.

\section{Current Study}

Although communication between partners during deployment has begun to be studied, there remain important areas for further examination, including how it may contribute to or alleviate relational turbulence. The current study examines couple deployment communication within the National Guard. We focus on multiple forms of communication, including video chat and social media. Through the lens of RTT, we examine couples' experiences with events that could contribute to relational turbulence.

\section{Methods}

Data for the current study were taken from a longitudinal study conducted by the authors on risk and resilience in National Guard service members and partners that spanned from pre-deployment to two years post-deployment. As part of this study, semi-structured interviews were conducted with 31 couples approximately three months after service members returned home. Given the focus on exploring resilience, couples selected for interviews were those who had been together in their intimate relationship prior to the deployment, had gone through the deployment together, and were committed to staying together at the time the deployment ended. Individuals in the study consisted of service members of the Army National Guard from a Midwestern state, and all service members had recently returned from an Afghanistan deployment in support of Operation Enduring Freedom. Almost all the service members sampled served in combat arms roles, significantly increasing the likelihood that they encountered direct combat. All activities related to participant recruitment, selection, and qualitative interviews were approved by the Institutional Review Boards of the study investigators as well as the US Army Medical 
Research and Materiel Command Human Research Protection Office (HRPO).

\section{Procedures}

Participants were invited to take part in the study at a reintegration event where service members and their partners gathered in order to receive support related to reintegration activities. Individuals were asked to volunteer to take part in qualitative interviews. Volunteers for these interviews were pre-screened to ensure they did not report high levels of suicidal ideation, heavy substance abuse, and or active intimate partner violence. If individuals reported these thoughts/ behaviors, they were not pursued for qualitative interview participation. Each member of the couple received an incentive of $\$ 50$ for completion of the interview. Interviews were conducted prior to the COVID-19 pandemic, and took place typically at participants' homes, or in some cases at mutually agreed upon, convenient public locations that ensured privacy. Couples were interviewed together first, and then individually. Each interview lasted between 90 and $120 \mathrm{~min}$. Interviewers began by reviewing the informed consent and answering questions. The interviews were informed by a semi-structured interview guide developed and piloted by the research team. Participants were asked questions regarding stressful events, coping resources, and adjustments they made, each in relation to deployment. Communication and connection during deployment was one major item that interested the study team and probes were explored along the way as these topics arose. Interview audio was recorded and transcribed at a later date. Data were stored on a secure server and participant confidentiality was maintained by using unique identifiers created by participants.

\section{Demographics}

The majority of service members were male (there was one female). Service members' ages ranged from over 50 years old $(n=1)$ to under 24 years $(n=4)$, with most of the sample falling between the ages of $25-30(n=7,22.6 \%)$ and $31-40(\mathrm{n}=11,35.5 \%)$. Partners' ages also ranged from over 50 years old $(n=1)$ to under 24 years $(n=5)$. Most of the partners were between 25 and 30 years old $(n=9$, $29 \%)$ and $31-40(n=11,35.5 \%)$. Regarding service member race, $6.5 \%$ were Black $(n=2), 3.2 \%$ were Hispanic $(n=1)$, $6.5 \%$ were Multiethnic $(n=2), 9.7 \%$ were "other" $(n=3)$ and $74.2 \%$ were White $(n=23)$. Partners' racial demographics included 3.2\% Black $(n=1), 9.7 \%$ Hispanic $(n=3), 3.2 \%$ Asian ( $(n=1), 9.7 \%$ Multiethnic $(n=3), 3.2 \%$ "other" $(n=1)$, and $74.2 \%$ White $(n=22)$. Most service members $(n=24$, $77.4 \%$ ) reported having at least some college, including 14 indicating that they had received a degree. For partners, the majority $(n=27,87.1 \%)$ had received at least some college education, 15 of which earned a degree. Seventeen of the 31 service members $(54.8 \%)$ indicated that they had been in the National Guard for over five years, of which ten reported that they had served 11 years or more. Over half of the service members $(n=16)$ had deployed previously, and four of those service members indicated that they had deployed multiple times. For the other half, this was their first deployment. The majority of the couples indicated having children living in their home $(55.3 \%)$, with one or two children being the most frequent $(n=13,42 \%)$ and five children $(n=2,6.5 \%)$ the most.

\section{Data Analysis}

Data analysis was guided by Thematic Analysis, with the goal of identifying, analyzing, and reporting on themes which emerged across the interviews (Braun \& Clark 2006). The following six Thematic Analysis steps were completed: (1) familiarization with the data in which the first author reviewed each interview transcript multiple times becoming deeply acquainted with each case; (2) open coding which generated a list of initial codes; (3) going over initial codes and triangulating them with other relevant data such as field notes and prior knowledge of National Guard couples, (4) checking codes against other codes and reducing the number of codes where there was redundancy, (5) developing themes by combining initial codes; (6) developing a narrative for the themes and their relation to each other (Braun \& Clark 2006). The first author took the lead with steps one and two, following which the first and second authors collaborated in steps three through six in order to identify and refine the findings. Saturation was achieved during the course of data analysis.

\section{Findings}

Data analysis yielded clear themes in what did and did not work well for couples during deployment. Findings below first highlight communication approaches that led to positive outcomes, and then illustrate negative communication approaches, as well as structural barriers to communication.

\section{Communication Approaches that Led to Positive Couple Outcomes}

\section{Planning and Established Expectations}

Couples who reported the best communication during the deployment reported taking the time to discuss how this would be prior to leaving. While no deployment scenario is predictable (e.g., there may not be a good internet connection), couples who reported communication success spent time considering these scenarios and how they might play 
out during the deployment. The following couple described their approach to planning that was typical of couples who were communicating regularly.

Interviewer: So were you able to talk like on key events, milestones, anniversaries, birthday's things like that?

Service Member: Well I actually had a...

Partner (finishing his sentence): ... had a game plan. I'd get off work and if we could jump on Skype or if we could talk on the phone or something you know it was nice. For milestones and things like that, like he sent me flowers on our anniversary... he planned ahead of time which I know some of the girlfriends and wives that I know of like they definitely didn't do that... He made a really big effort to maintain those traditions.

\section{Routine and Regularity}

Couples reported that communication plans went well when they included predictability of some kind. Although this was not always a good thing, for the most part, having the expectation that contact would be made was a reassuring presence for the couples. One partner describes how this regularity helped:

Partner: Almost every night, we had a date while he was deployed. I don't know how partners got through deployment before Skype but, Skype was amazing for us... He would always get up early before he went to work and we would talk. So, my daughter and I would have that time with him.

\section{Addressing Problems in the Moment}

Deployment changes both service members and partners. This was true for the couples we interviewed. While there are limits to communication on a deployment, partners who appeared to be doing the best in reintegration were also the couples who talked regularly and addressed problems in their lives as they came up. This process allowed for them to keep up with changes in each other and in their lives. When too many things were kept secret, the adjustment postdeployment was more difficult. One partner describes this as follows:

Partner: We talk a lot and we try to keep it really open even stuff that is not pleasant, but we talk... I think that is the biggest thing you just have to talk and be honest and be willing to be offended without being offended. I think going into this one was so different because we were prepared...We knew like the way his brain absorbs stress and we were kind of prepared for some of the PTSD. So communication I think made it easier this time like from the very beginning.

\section{Flexibility and Realistic Expectations}

Deployment was unpredictable, and communication was often disrupted. Couples needed to be prepared for this. The following couple describes this required flexibility:

Partner: He called me more than I actually thought he was going to which is better, but I kind of knew that if he didn't call me for a couple days or whatever, I either knew he was on the road or, you know, I know enough about the military to know that he probably had a mission.

Service Member: We had some blackout communications, couldn't call when the stuff was down, whatever. Partner: So, I knew he would call me eventually. Service Member: So, you roll with it.

\section{Extra Effort}

Communication was most meaningful for partners when service members demonstrated intentionality and effort. Most partners we interviewed wanted to hear from their service member as much and as often as possible. Some service members made a big effort to communicate, while others did not. When the effort was made, communication home went well and partners felt loved, secure, and cared about. However, for some service members, calling home was considered an added chore to a stressful deployment, and they avoided this. In some cases, this left a partner feeling hurt and uncared about. The following two quotes represent two service members making an incredible effort to communicate home each day.

Service Member: When I Skyped the most was at like 3 o'clock in the morning so, you know, it's go to bed at midnight, get back up at 3 o' clock, you know, Skype for an hour, go back to bed for two hours, you know, start the day over again. That's just what you have to do when you got several kids at home...

And a second couple:

Interviewer: Was it hard for you though to get up early every day and call her and talk to her?

Service Member: No, no. I just made it part of my routine, that's just what I did, yeah, I got up in the morning, went to breakfast and Skyped and then I went to work. Now they (other service members) all had the same opportunity whether they want to get out of bed and do that that's a different story but, yeah, and I think 
that can be some of it too it's just that whether you're going to put your priorities there and do it or not....

\section{Consistency and Connection}

Consistent communication was particularly important in keeping couples emotionally connected through a lengthy and anxiety provoking deployment. For some couples, being involved in the seemingly mundane day-to-day activities of one another's lives seemed to offer peace of mind and constancy amidst the uncertainty of deployment. This sustained communication was also helpful in allowing couples to reconnect quickly once the deployment ends. The following couple described just 'hanging out' on Skype:

Partner: There were times at work where I mean he would just want to be on [Skype] so he could see normal life. So, I would just have him on a computer he could just see my office.....and he could see coworkers and stuff...but it was just quiet where I was working and he was just doing his thing.

Service Member: I'd kind of read news on the line and have that on the sidebar.

Partner: It was just us hanging out but not really even having to talk. I mean, so it was nice. We did, we Skyped an awful lot I think.

Another partner described a similar ongoing connection which provided peace of mind:

Partner: We had constant communication...he would get up in the morning to get ready for his shift and he would turn on his Skype and we'd be able to Skype before I went to bed, so I could go to bed with ease knowing that he was okay and I got to see his face and then when he worked nights we would email back and forth most of the day... I'd fill him in on everything and I never made big choices without letting him know... I even went down to what color I should paint the living room... so he knew what he was coming home to.

\section{Communication Innovation}

Some couples used innovative ways of communicating and staying connected, ranging from daily emails to sending care packages, jokes, or news clips. One couple had an innovative way of sharing stressful experiences endured during communication through the use of a joint diary. The following quote captures this process:

Partner: I think because we were communicating the whole time and not just via email and letters and things like that, we really were able to continue to communicate.

Service Member: We have a joint diary that she communicates well in.

Partner: Because when he was gone, it was the one thing that I did for myself. I'm like, 'Okay, I'm really stressed out but, I'm not going to worry him about it. So, I'm just going to write it in my own little journal.' And then, I gave it to him when he returned after a couple of weeks...So that he's like, 'Oh, I didn't know that it was quite like that for you.' So, it was an eye opener for him, and then, we just kind of kept one together because sometimes you just need to get something off your chest.

\section{Communication Approaches That Led To Negative Emotions}

In the themes that arose out of the interviews, there were clear communication approaches that did not alleviate couples' experiences of uncertainty. Some of these are the antithesis of the strengths described above and include lack of planning or routine regarding communication, or little effort made by the service member. These will not be described again in this section other than to say, these led to poor communication. There were other negatives as well.

\section{Observation of Home Life}

In some cases, while communicating with home, service members witnessed aspects of home life that brought up strong emotions. This included family members having fun, family members being stressed out, or simple things that made the service member miss home. Mostly, these were negative for the service member and required some discussion about how to manage these things when they occurred. In the following excerpt, a service member describes negative feelings associated with seeing his grown children sitting around a fire. This brought up feelings of longing and a sense of missing out:

Service Member: The kids and family came onto Skype a couple of times and they're partying and drinking beer, and the computer was with them in the driveway.

Partner: Yeah, and the oldest one he came over, he thought it would be fun to Skype dad and we were having a little camp fire and that they would have the Skype going while they had a beer and were talking to him. He was like, 'Yeah, I don't enjoy this so much!' 


\section{Passive Social Media Use}

Although social media allowed couples to stay connected, in some cases where it was used passively (i.e. observing others' posts and communication) it led to hurt feelings. Some partners witnessed service members and their partners talking online extensively, which when compared to their communication with their own service member led to feelings of insecurity and not being cared about, as this partner described:

Partner: Social media is a challenge to be honest with this because I would see guys because I'm friends with his friends in his squad, guys that I know he's with and they're like on Facebook posting or chatting and I'm like 'How come they're on there and where are you?... Why haven't I heard from you in three days?' So that was tough for us. That was really tough for us.

Some service members learned secrets or information on social media that they did not hear from their partner, but which made them feel like their partner was not telling them everything, as is described in this exchange between a couple:

Service Member: Well there are things that I really don't want to know about that are going on at home. I don't want to know that my aunt and my sister and everybody else are having arguments. I needed to be more focused on other things I don't need to be focused on the stuff going on back here, especially if its stuff that I could wait and find out later.

Partner: And the problem with that was on your end because you couldn't stay off of Facebook and that is where you found out your information not because I told you.

Service Member: Well some of it.

Partner: I did try to filter some of that stuff for you and then you would find out and then ask me, but because I don't do Facebook I don't know that information. But part of that was you seeking information and finding stuff you didn't want to know.

Generally, the passive use of social media was negative and left the partner at home with bruised feelings and service members feeling like they were kept in the dark by their partner.

\section{Shallow Communication}

Another negative described by couples regarding communication was the need to protect each other from aspects of their experiences of deployment. This in some cases was because of the military restricting what could be talked about, and in other cases because the partner wanted to protect the service member from difficulties occurring at home. In all these cases, these scenarios led to shallow communication or in couples not knowing about their loved one's daily stressors. Attempting not to stress each other out during deployment, or avoiding topics until a later time, created other problems down the line when couples reintegrated. The following quotes capture this experience:

Service Member: ... for six months we didn't talk about any kind of real problems because we knew we just had a few minutes to talk and we were just like, 'Okay, how many days left?' and we didn't really have the opportunity to talk about any of this stuff.

These things were particularly difficult if they were a big issue or if the couple did not want to fight or disagree while on deployment (normal aspects of any relationship). War was not considered an appropriate context to address more intense or difficult relationship issues - they were put on hold. At times this was due to a lack of privacy or a bad connection, but mostly it was because couples did not want to be upset on a deployment. The following couple captured this dilemma:

Service Member: being newly married and hearing about the different things going on back home, being worried about her and not being able to do anything about it... We tried to make sure that we were being there for each other but then also not... we tried to keep things a little bit lighter to make sure that we ended on a good note because we didn't know necessarily the next time that we would end up being able to talk so we didn't want the last conversation that we had to be the one that was an argument or something like. We figured that if there was something that really needed to be discussed it would be tabled until we got back if it was a big enough issue. We both realized if there were big issues it wasn't going to be something that we were going to solve from 6000 miles away.

\section{Structural Barriers that Led to Negative Outcomes}

Finally, there were structural issues that occurred during deployment which were largely out of the control of the couple, but which limited or prevented communication.

\section{Technology Issues}

A portion of couples in this study struggled with uncertainty due to technological issues. For some, these issues were due to the inconsistent technology found in a war zone. For others, communication problems related to poor equipment back home or lack of knowledge about how to operate programs such as Skype. This invariably led to frustration, 
stress, and strain. The following couple captures the essence of this theme:

Partner: The most frustrating thing for me honestly was that we couldn't get Skype to work... Overseas we could get it to work a couple of times. We could never get it to work when he was down in Mississippi and that just seemed ridiculous that you know technology wasn't there for us.

Another couple described how problems with technology prevented them from having needed, meaningful conversations about their relationship:

Partner: And the Wi-Fi would cut out all the time. You would be saying something and you'd have to call back and it was a huge hassle.

Service Member: ...there was never really any kind of time to talk about deeper relationship-type issues.

\section{Communication Blackouts}

Communication blackouts, which occur on deployment when someone is hurt or when there is substantial fighting, were a particular source of anxiety and even panic for partners. We were moved when we heard about the intensity of these issues and how they affected partners back home. While these incidents may be outside of military policy or control, they nevertheless had a detrimental effect on partners. One partner's anxiety was still palpable in the following interview when describing her experience during a communication blackout that had happened months ago:

Service Member: One of the days that we had left to resupply, something bad happened... of course she had no idea what was going on. Uh, everything blacks out... all communications are blacked out, so. I had no way of letting her know I'm okay... It was a difficult situation.

Partner: ...it was quite devastating... I had gone to a wedding with one of our best friends... I was alone in my hotel room and his dad texted me and says, 'Did [service member] get hit?' And I'm like, 'You woke me up with that?' And I'm like, 'What are you talking about?' And he goes, 'His base, did he get hit? You know a guy's down. You know a certain guy's hurt.' I'm freaking out at this point... so I'm trying to watch the news, you know... and I'm, I'm just like scared to death. I don't know who to call, who to get a hold of and finally I'm just like... I'm just calling this FRG leader... I just called her hysterically like crying like 'You gotta answer some questions.' No one told me the protocol before they left... if something happens that there is a 24-hour period before it ever hits the news, because they do contact the partners first and they'll find you if you're not at home, thankfully. No one told me the protocol. And so I'm freaking out...

Another partner described an incredibly anxiety provoking ordeal after her husband was injured in a bomb blast. This anxiety was caused by the bomb blast and the injury, but also because she received incomplete communications about the situation and the extent of his injury.

Partner: I got a phone call from his buddy who was in the field as well saying [service member] got hurt... It was basically straight out of a movie because the phone was all static and he couldn't give me a lot of details. It was a ten-second conversation and then the line dropped and then he called back basically saying again [service member] got hurt... that he hurt his back pretty bad. I asked him if he still had movement of his legs and were his legs still intact and all of that. His friend wouldn't tell me he just said '[service member] will call you when he can' and then the line died. So I immediately drove over to his family's house, filled them in and then we just sat there and waited by the phone. The military called me 18 hours after his friend had called me basically saying '[service member] suffered a back injury and we're not sure of the extent of his injuries but stay tuned' and that is all I got. Another 12 hours after that [service member] Facebooked me saying that he was okay and that he was in a hospital and basically went through his injuries, what had happened, and then told me he would be in touch. The day after that the military called again basically saying just wanted to check in but we have no information for you... that was pretty much the last communication from them. I heard from [service member] that he was going to be flying home through Germany to Walter Reed so I immediately began making plans of transferring down there locally with my job... It was quite the journey... it was grueling.

The above two scenarios capture the intensive anxiety for partners back home. These are traumatic experiences that invoke surges of anxiety, emotion, fear, and tears. These partners were profoundly affected by these types of events, and they were not the only ones.

\section{Discussion}

Our findings indicate that relational turbulence can occur for military couples during deployment through various contributors to relational uncertainty. Some partners found themselves questioning their partner's commitment to their relationship, such as through comparison to another couple's communication on social media. Relational 
uncertainty was also manifest as ambiguity regarding what type of information to share, and trying to decide how to best meet the needs of their partner. Uncertainty likewise occurred due to external circumstances, such as the breakdown of technology and communication blackouts. Many couples reported experiencing emotional markers of relational turbulence, including anger, sadness, fear, and jealousy (Knobloch \& Theiss, 2014), due to the uncertainty that communicating during deployment presented. However, despite the potential for relational turmoil due to the uncertainty of deployment, many couples demonstrated strength, unity, and resilience through their intentional approaches to communication. Below we discuss these findings, and suggest how couples can make a communication plan together which considers each other's needs and the realities of deployment in order help mitigate relational turbulence.

\section{Communication Planning}

Communication was positive for couples when it was planned out in advance with an intentional discussion of potential obstacles and barriers. This finding is consistent with Sayers and colleagues' (2018) assertion that discussion of communication preferences prior to deployment is important for couples, and may even lead to better post-deployment relationship outcomes. As such, couples should develop realistic expectations around communication with built-in flexibility. Conversations that cover multiple possible scenarios prior to the deployment can go a long way to establish these guidelines. For couples who had been through this before, it was easier; for other couples, they were learning by trial and error. We advocate for all couples to have a communication plan prior to deploying. Being prepared is an important part of the military culture and when couples are prepared, their time apart is much more connecting. These plans need to include preparing for the use of technology, as well as a predictable routine.

Of note is that these routines should be negotiated in a way that meets the needs of both parties, which may include communication frequency or purpose (Sayers et al., 2018). Some service members prefer not to communicate regularly, which may in part reflect an effort to avoid distractions while deployed. However, couples who used this approach report more relationship strain and disconnection post deployment (Durham, 2010). On the contrary, regular communication may help prevent partners back home from feeling isolated, unloved, and unimportant. Having couples achieve consensus on these things prior to leaving is an important part of helping communication to go well on the deployment.

\section{Active and Open Communication}

Couples seem to do better when they can effectively address problems and hear about each other's stress as it occurs. This finding is in keeping with past studies which report that information withholding during deployment can leave both soldiers and at-home partners feeling less connected and is linked to worse partner outcomes (Baptist et al., 2011; Carter \& Renshaw 2016; Joseph \& Afifi, 2010). However, there is also evidence that in some couples, the withholding of certain information, such as traumatic events or problems at home is preferred, with the goal of protecting their partner (Sayers et al., 2018). In the current study, both service members and at-home partners altered the content of their conversations at times in order to share what they perceived to be in the best interest of their partner. Despite the intention to save their partner from undue stress or distraction, some attempts to withhold stressful information appeared to inadvertently weaken the depth of the relationship between partners. This indicates the need for balance between openness and withholding of stressful or confidential information; a balance which is ideally determined by each couple for themselves.

We propose, that when feasible, couples work to share as openly and honestly as possible. In some cases, where couples are unsure about this, they may benefit from coaching from a relationship expert prior to deployment so that couples can resolve problems more readily and communicate support with each other. Additionally, couples should discuss together prior to deployment any topics or situations in which full disclosure will not be possible or is not preferred, whether to maintain operational security or to reduce unnecessary burden on the soldier.

\section{Social Media Rules and Guidelines}

Our findings indicate that social media can be harmful during deployment and should be used with discretion. Social media served multiple functions, including the option for service members and partners to easily check in on one another. However, in some cases it was not helpful and brought up hurt and jealous feelings. Social media use is known to create relational conflict (Fox \& Moreland, 2015) and jealousy, especially in couples in long-distance relationships (Billedo et al., 2015). It is also linked to lower perceived social support in deployed soldiers when used maladaptively (Skopp et al., 2018). Couples in our study reported similar experiences. Perhaps the passive use of social media to check in on one's partner is a manifestation of relational uncertainty, rather than an intentional attempt to connect with one's partner. Although some military spouses report the benefits of social media use during a soldier's deployment, they emphasize the importance of avoiding drama and maintaining openness (Rea et al., 2015. Additionally, 
successful social media use by military spouses did not always involve direct communication with the service member, but rather using it as a resource to connect with others, such as through support groups (Rea et al., 2015). It seems that it is best for couples to discuss social media usage in advance of the deployment, including if and how they would like to use social media, and that they plan specifically how it can be used in active, constructive ways in order to stay connected.

\section{Communication Blackouts}

Finally, communication blackouts and incomplete communication caused substantial distress, particularly for partners back home. These partners spent hours, and sometimes days, with the panic that something terrible had happened to their loved one. In at least one case, this distress was heightened by a partner's lack of understanding of blackout communication protocol. We recommend that the military make special efforts to educate service members and their partners on black out communication protocol prior to deployment. Further, we suggest couples create a plan together of how the at-home partner can respond in the event of a blackout (i.e. reach out to a Family Readiness Group [FRG] leader, family or friends, etc.) in order to help provide the partner with needed support until communication is restored.

\section{Implications}

The findings of this study have meaningful implications for National Guard couples. Despite the uncertainty that deployment brings, our findings highlight the value of couples intentionally planning how to stay connected throughout their time apart. As couples share their needs with one another and plan for how these needs can be met through communication during deployment, they may respond more resiliently to the challenges of deployment. Therapists in particular may lend support to military couples during the pre-deployment phase by helping them discuss and create a communication plan. Specifically, therapists may help both partners articulate their communication needs and expectations to one another. These communication plans should be couple-specific and should consider factors such as sensitive topics for the couple and any history that may make certain situations particularly triggering for either the service member or at-home partner. Therapists could also encourage couples to role play various scenarios in which communication breakdowns may occur. In this situation the therapist could help couples identify the negative emotions that may result, and help with the development of active coping strategies in response to these emotions. The military can likewise ensure that couples plan in advance for communication, such as through providing information and mentoring materials about what to expect and how to navigate communication challenges. A collaborative effort between therapists and military personnel may be particularly effective in assisting these couples.

\section{Limitations and Future Directions}

Like any study, this study has limitations. The couples interviewed were all from the National Guard in one Midwestern state, whose experiences may be different than those serving in active duty or in other branches of the military throughout the country. The couples who were interviewed all volunteered, and as such their experiences may have been different to non-volunteers. Thus, these findings should be interpreted with caution, as they are not generalizable beyond the population surveyed. However, we believe that the principles considered would be important for all deploying couples to consider. Future research should likewise be devoted to exploring the potential nuances of deployment communication in underrepresented couples (e.g. racial/ethnic minorities and non-heterosexual couples).

Although the current study provides valuable insight into how couples can achieve effective communication during deployment, there remains more to be done. As the qualitative findings suggest that communication planning is beneficial for couples, future studies should quantitatively explore how this planning may relate to mental health and relational outcomes for soldiers and their partners. Due to the relatively limited understanding about the implications of social media use during deployment, ongoing research should explore the impact of social media on couples during deployment, and specifically how it can serve as a benefit, rather than a hindrance. It would also be useful to study other contextual factors surrounding deployment communication more in-depth, such as how communication may vary based on the number of deployments a soldier has served or how it may be different for active duty soldiers.

Funding This work was supported by the Office of the Assistant Secretary of Defense for Health Affairs through the Psychological Health/Traumatic Brain Injury Research Program under Award No. W81XWH-12-1-0419 and 0418 (Blow, PI; Gorman, Partnering PI).

\section{Declarations}

Conflict of interest The authors have no relevant financial or non-financial interests to disclose.

\section{References}

Baptist, J. A., Amanor-Boadu, Y., Garrett, K., Nelson Goff, B. S., Collum, J., Gamble, P., Gurss, H., Sanders-Hahs, E., Strader, L., \& Wick, S. (2011). Military marriages: The aftermath of Operation 
Iraqi Freedom (OIF) and Operation Enduring Freedom (OEF) deployments. Contemporary Family Therapy, 33(3), 199-214. https://doi.org/10.1007/s10591-01191626

Billedo, C. J., Kerkhof, P., \& Finkenauer, C. (2015). The use of social networking sites for relationship maintenance in long-distance and geographically close romantic relationships. Cyberpsychology, Behavior, and Social Networking, 18(3), 152-157. https:// doi.org/10.1089/cyber.2014.0469

Braun, V., \& Clarke, V. (2006). Using thematic analysis in psychology. Qualitative Research in Psychology, 3(2), 77-101. https://doi.org/ 10.1002/jts.20649

Carter, S., Loew, B., Allen, E., \& Stanley, S. M. (2011). Relationships between soldiers' PTSD symptoms and spousal communication during deployment. Journal of Traumatic Stress, 24(3), 352-355. https://doi.org/10.1002/jts.20649

Carter, S. P., \& Renshaw, K. D. (2016). Spousal communication during military deployments: A review. Journal of Family Issues, 37(16), 2309-2332. https://doi.org/10.1177/0192513X14567956

Caska, C. M., \& Renshaw, K. D. (2011). Couple's communication during deployment: Associations with PTSD, social support, and relationship satisfaction. In D. K. Snyder (Chair), Emerging evidence regarding post-traumatic stress disorder and relationship functioning in OEF/OIF combat veterans. Symposium conducted at the annual meeting of the Association of Behavioral and Cognitive Therapies, Toronto, Canada.

Cigrang, J. A., Talcott, G. W., Tatum, J., Baker, M., Cassidy, D., Sonnek, S., Snyder, D. K., Balderrama-Durbin, C., Heyman, R. E., \& Smith Slep, A. M. (2014). Intimate partner communication from the war zone: A prospective study of relationship functioning communication frequency, and combat effectiveness. Journal of Marital and Family Therapy, 40(3), 332-343. https://doi.org/10. 1111/jmft.12043

Durham, S. W. (2010). In their own words: Staying connected in a combat environment. Military Medicine, 175(8), 554-559. https:// doi.org/10.7205/MILMED-D-09-00235

Fox, J., \& Moreland, J. (2015). The dark side of social networking sites: An exploration of the relational and psychological stressors associated with Facebook use and affordances. Computers in Human Behavior, 45(4), 168-176. https://doi.org/10.1016/j.chb. 2014.11.083

Glaser, B., \& Strauss, A. (1967). The discovery of grounded theory. Aldine.

Joseph, A. L., \& Afifi, T. D. (2010). Military wives' stressful disclosures to their deployed husbands: The role of protective buffering. Journal of Applied Communication Research, 38(4), 412-434. https://doi.org/10.1080/00909882.2010.513997

Knobloch, L. K., Ebata, A. T., McGlaughlin, P. C., \& Ogolsky, B. (2013). Depressive symptoms, relational turbulence, and the reintegration difficulty of military deployment following wartime deployment. Health Communication, 28(8), 754-766. https://doi. org/10.1080/10410236.2013.800440

Knobloch, L. K., Knobloch-Fedders, L. M., \& Yorgason, J. B. (2018). Communication of military couples during deployment predicting generalized anxiety upon reunion. Journal of Family Psychology, 32(1), 12-21. https://doi.org/10.1037/fam0000344

Knobloch, L. K., \& Theiss, J. A. (2014). Relational turbulence within military couples during reintegration following deployment. In S. MacDermid Wadsworth \& D. Riggs (Eds.), Military deployment and its consequences for families. Risk and resilience in military and veteran families. Springer.

Martin, J., Ghahramanlou-Holloway, M., Lou, K., \& Tucciarone, P. (2009). A comparative review of US military and civilian suicide behavior: Implications for OEF/OIF suicide prevention efforts. Journal Mental Health Counseling, 31(2), 101-118. https://doi. org/10.17744/mehc.31.2.a6338384r2770383

Merolla, A. J. (2010). Relational maintenance during military deployment: Perspectives of wives of deployed US soldiers. Journal of Applied Communication Research, 38(1), 4-26. https://doi.org/ 10.1080/00909880903483557

Moelker, R., \& van der Kloet, I. (2006). Military families and the armed forces. In G. Caforio (Ed.), Handbook of the sociology of the military (pp. 201-223). Springer.

Ponder, W. N., \& Aguirre, R. T. (2012). Internet-based spousal communication during deployment: Does it increase post-deployment marital satisfaction? Advances in Social Work, 13(1), 216-228. https://doi.org/10.18060/1867

Rea, J., Behnke, A., Huff, N., \& Allen, K. (2015). The role of online communication in the lives of military spouses. Contemporary Family Therapy, 37(3), 329-339. https://doi.org/10.1007/ s10591-015-9346-6

Sayers, S. L., Barg, F. K., Mavandadi, S., Hess, T. H., \& Crauciuc, A. (2018). Deployment communication: Underlying processes and outcomes. Journal of Family Psychology, 32(1), 3-11. https://doi. org/10.1037/fam0000339

Skopp, N., Alexander, C., Durham, T., \& Scott, V. (2018). Positive and negative aspects of Facebook use by service members during deployment to Afghanistan: Associations with perceived social support. Psychology of Popular Media Culture, 7(3), 297-307. https://doi.org/10.1037/ppm0000123

Solomon, D. H., Knobloch, L. K., Theiss, J. A., \& McLaren, R. M. (2016). Relational turbulence theory: Explaining variation in subjective experiences and communication within romantic relationships. Human Communication Research, 42(4), 507-532. https:// doi.org/10.1111/hcre. 12091

Solomon, D. H., \& Theiss, J. A. (2011). Relational turbulence: What doesn't kill us makes us stronger. In W. R. Cupach \& B. H. Spitzberg (Eds.), The dark side of close relationships II (pp. 197-216). Routledge

Theiss, J. A., \& Knobloch, L. K. (2013). A relational turbulence model of military service members' relational communication during reintegration. Journal of Communication, 63(6), 1109-1129. https://doi.org/10.1111/jcom.12059

Warner, C. H., Breitbach, J. E., Appenzeller, G. N., Yates, V., Grieger, T., \& Webster, W. G. (2007). Division mental health in the new brigade combat team structure: Part I. Predeployment and Deployment. Military Medicine, 172(9), 907-911. https://doi.org/10. 7205/milmed.172.9.907

Wong, L., \& Gerras, S. R. (2006). CU@ the FOB: How the forward operating base is changing the life of combat soldiers. DIANE Publishing.

Publisher's Note Springer Nature remains neutral with regard to jurisdictional claims in published maps and institutional affiliations. 